Case Report

\title{
Orthodontic extrusion with palatal circumferential supracrestal fiberotomy improves facial gingival symmetry: a report of two cases
}

\author{
Naoto Yoshinuma ${ }^{1,2)}$, Shuichi Sato ${ }^{1,2)}$, Noritsugu Makino'), Yuichi Saito ${ }^{1,2)}$ \\ and Koichi Ito $^{1,2)}$ \\ ${ }^{1)}$ Department of Periodontology, Nihon University School of Dentistry, Tokyo, Japan \\ ${ }^{2)}$ Division of Advanced Dental Treatment, Dental Research Center, Nihon University School of Dentistry, \\ Tokyo, Japan
}

(Received 20 June and accepted 20 August 2009)

\begin{abstract}
Discrepancy in the labial gingival margin of the maxillary incisors poses a major aesthetic problem. Orthodontic extrusion can improve the aesthetic problem caused by gingival margin levels before restoration. In these case reports, orthodontic extrusion was performed with palatal circumferential supracrestal fiberotomy for improving the discrepancy of the labial gingival margin. Two years postoperatively, the position of the tooth and gingival margin remained stable. Orthodontic extrusion with palatal circumferential supracrestal fiberotomy was effective in improving the discrepancy of the labial gingival margin of maxillary incisors. (J Oral Sci 51, 651-654, 2009)
\end{abstract}

Keywords: orthodontic extrusion; gingival margin; fiberotomy; intrasulcular incision; discrepancy.

\section{Introduction}

Discrepancy in the labial gingival margin of the anterior maxillary area presents a major aesthetic problem. When gingival margin discrepancies are present, orthodontic extrusion can improve the aesthetic problems of gingival

Correspondence to Dr. Shuichi Sato, Department of Periodontology, Nihon University School of Dentistry, 1-8-13 Kanda-Surugadai, Chiyoda-ku, Tokyo 101-8310, Japan

Tel: +81-3-3219-8107

Fax: +81-3-3219-8349

E-mail: sato-su@dent.nihon-u.ac.jp marginal levels before restoration (1). The extrusive tooth movement leads to coronal positioning of the intact connective tissue attachment, but orthodontic extrusion reverses the osseous architecture around the tooth being extruded. Consequently, osseous funneling or lipping results around the extruded root, which is leveled surgically to restore the physiologic form. Occasionally, the extruded teeth are splinted with adjacent teeth to prevent relapse (2), but splinting tends to promote plaque accumulation, leading to caries and gingival inflammation.

Circumferential supracrestal fiberotomy (CSF) prevents reversal of the osseous architecture and the relapse of extruded teeth (3). This technique consists of inserting a scalpel into the gingival sulcus and severing the epithelial attachment surrounding the extruded teeth (4). However, labial fiberotomy can cause gingival recession (5). We performed partial fiberotomy to improve the aesthetic problem treated by orthodontic extrusion. In these case reports, we performed orthodontic extrusion with CSF on the palatal half of the root to improve the discrepancy in the labial gingival margin. Here, we present the results after 2 years without splinting.

\section{Case 1}

\section{Case Reports}

A 24-year-old woman was referred for aesthetic correction of a labial gingival margin discrepancy at the maxillary left central incisor (tooth \#21). The tooth had normal mobility and clinical probing depths of 1 to $2 \mathrm{~mm}$. She had no malocclusion. The tooth was erupted $3 \mathrm{~mm}$ by orthodontic extrusion to improve the labial gingival 
margin level (Fig. 1). One millimeter of extrusion was achieved in 4 weeks using a 0.014-inch nickel titanium wire for aesthetic reasons (the difference in the incisal edge) and to prevent mesial rotation of the adjacent teeth. Following the active period of extrusion (Fig. 2), the teeth were maintained for 8 weeks using the same fixed orthodontic appliance. During orthodontic extrusion, an intrasulcular incision was made through the junctional epithelium and attachment apparatus on the palatal half

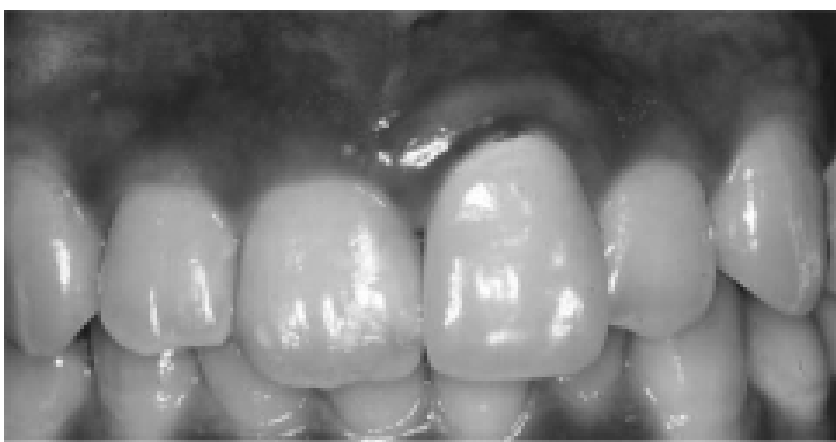

Fig. 1 Preoperative photograph of the maxillary left central incisor (tooth \#21).

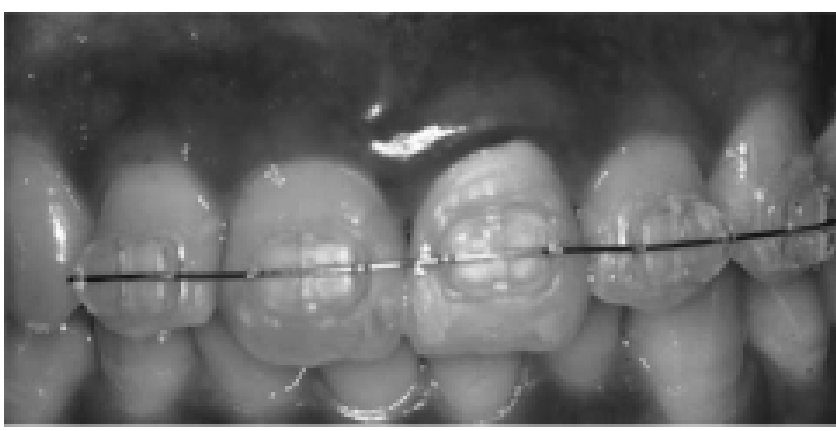

Fig. 2 Orthodontic brackets are attached to the crowns.

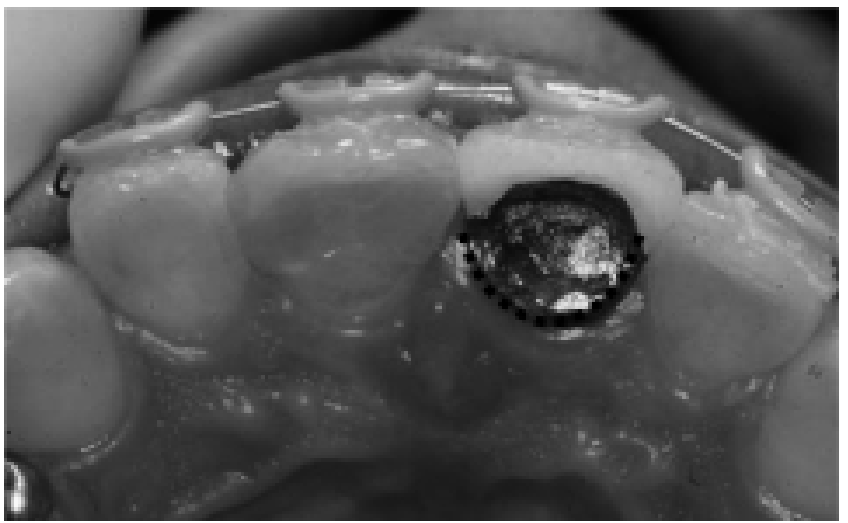

Fig. 3 Palatal fiberotomy. The dotted line is the incision line. of the root every 2 weeks using a scalpel and a \#11 blade (Fig. 3). This procedure was repeated three times. The final crown was inserted without splinting to adjacent teeth. Two years postoperatively, the position of the tooth and gingival margin remained stable (Fig. 4), and the tooth had normal mobility.

\section{Case 2}

A 41-year-old woman was referred for aesthetic correction of a labial gingival margin discrepancy in the maxillary right lateral incisor (tooth \#12; Fig. 5). The tooth had normal mobility and clinical probing depths of 1 to $2 \mathrm{~mm}$. She had no malocclusion. The tooth was erupted $2 \mathrm{~mm}$ by orthodontic extrusion to improve the labial gingival margin level using the same procedure as in case 1 , although the fiberotomy at the palatal half of the root was repeated twice. After treatment, the discrepancy in the labial gingival margin improved. The final crowns were inserted without splinting to adjacent teeth. Two years postoperatively, the position of the gingival margin remained stable (Fig. 6). The width of the keratinized

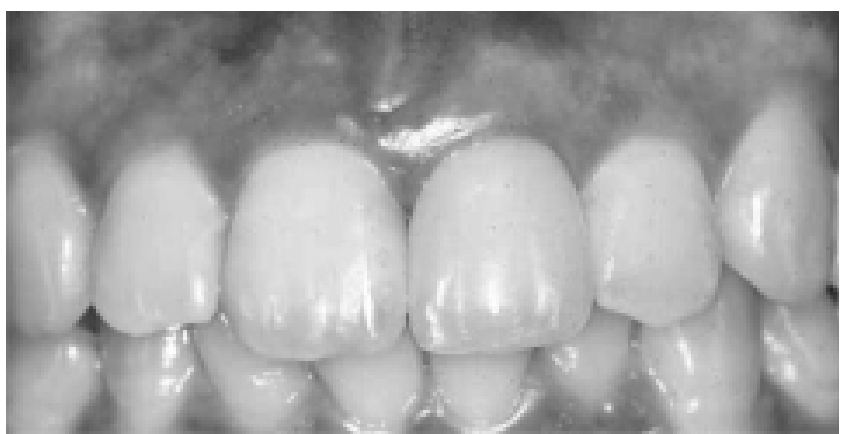

Fig. 4 Postoperative photograph 2 years after inserting the final crown. Note the symmetry of the gingival margins.

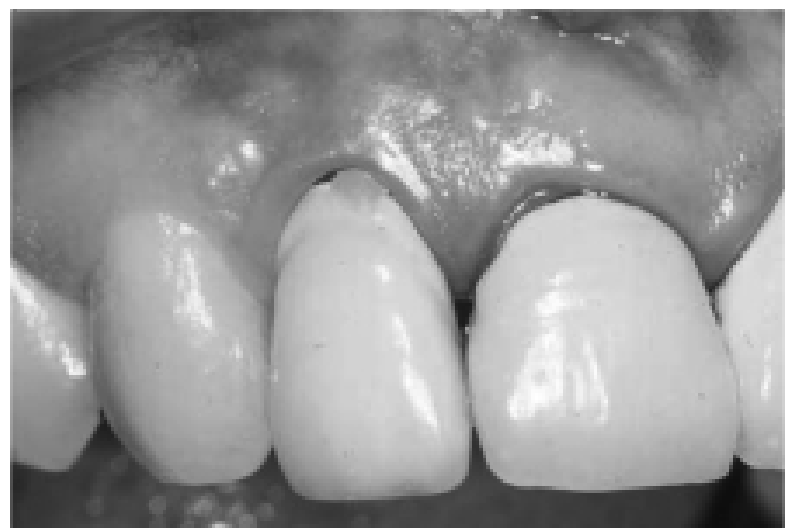

Fig. 5 Preoperative photograph of the maxillary right lateral incisor (tooth \#12). 
gingiva increased $2 \mathrm{~mm}$ as the tooth was extruded. The tooth had normal mobility and clinical probing depths of 1 to $2 \mathrm{~mm}$.

\section{Clinical and radiographic evaluation}

The clinical probing depth (PD) was measured on the midfacial aspect and it did not change during the followup period.

The keratinized gingiva (KG; the distance between the margin of the gingiva and mucogingival junction) was measured in the midfacial aspect. The KG increased by 3 $\mathrm{mm}$ in case 1 and $2 \mathrm{~mm}$ in case 2 at insertion of the final crown and remained unchanged over the 24 months following insertion of the final crown.

The distance between the root apex and the reference line between the apices of the adjacent roots was measured. The initial distance was $2 \mathrm{~mm}$ in both cases. This distance changed to 5 and $4 \mathrm{~mm}$, respectively, at insertion of the final crown. The distance did not change in the 24 months following insertion of the final crown (Fig. 7 and Table $1)$.

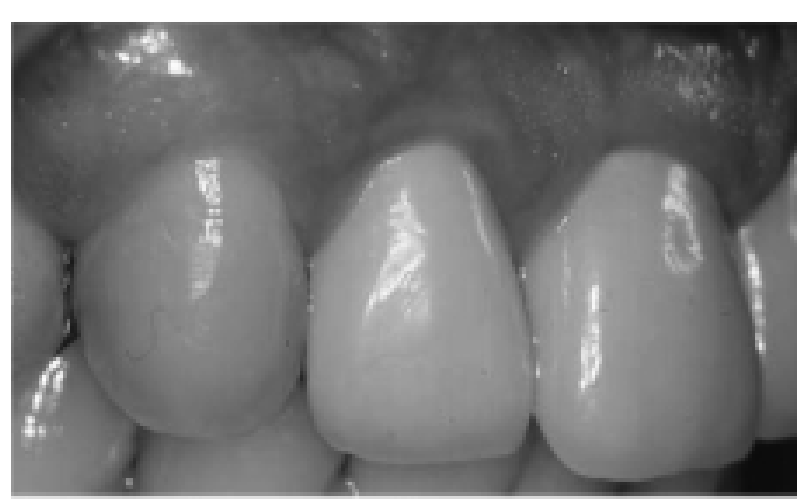

Fig. 6 Postoperative photograph 2 years after inserting the final crowns. Note the symmetry of the gingival margins.

Table 1 Measurements of clinical and radiographic parameters

\begin{tabular}{lcccccc}
\hline & \multicolumn{3}{c}{ Case 1 } & \multicolumn{3}{c}{ Case 2 } \\
\cline { 2 - 7 } & Initial & $\begin{array}{c}\text { Insertion } \\
\text { of crown }\end{array}$ & 24 months & Initial & $\begin{array}{l}\text { Insertion } \\
\text { of crown }\end{array}$ & 24 months \\
\hline PD $(\mathrm{mm})$ & 1 & 1 & 1 & 1 & 1 & 1 \\
KG $(\mathrm{mm})$ & 3 & 6 & 6 & 3 & 5 & 5 \\
Apex-Reference & 2 & 5 & 5 & 2 & 4 & 4 \\
Line $(\mathrm{mm})$ & & & & & & \\
\hline
\end{tabular}
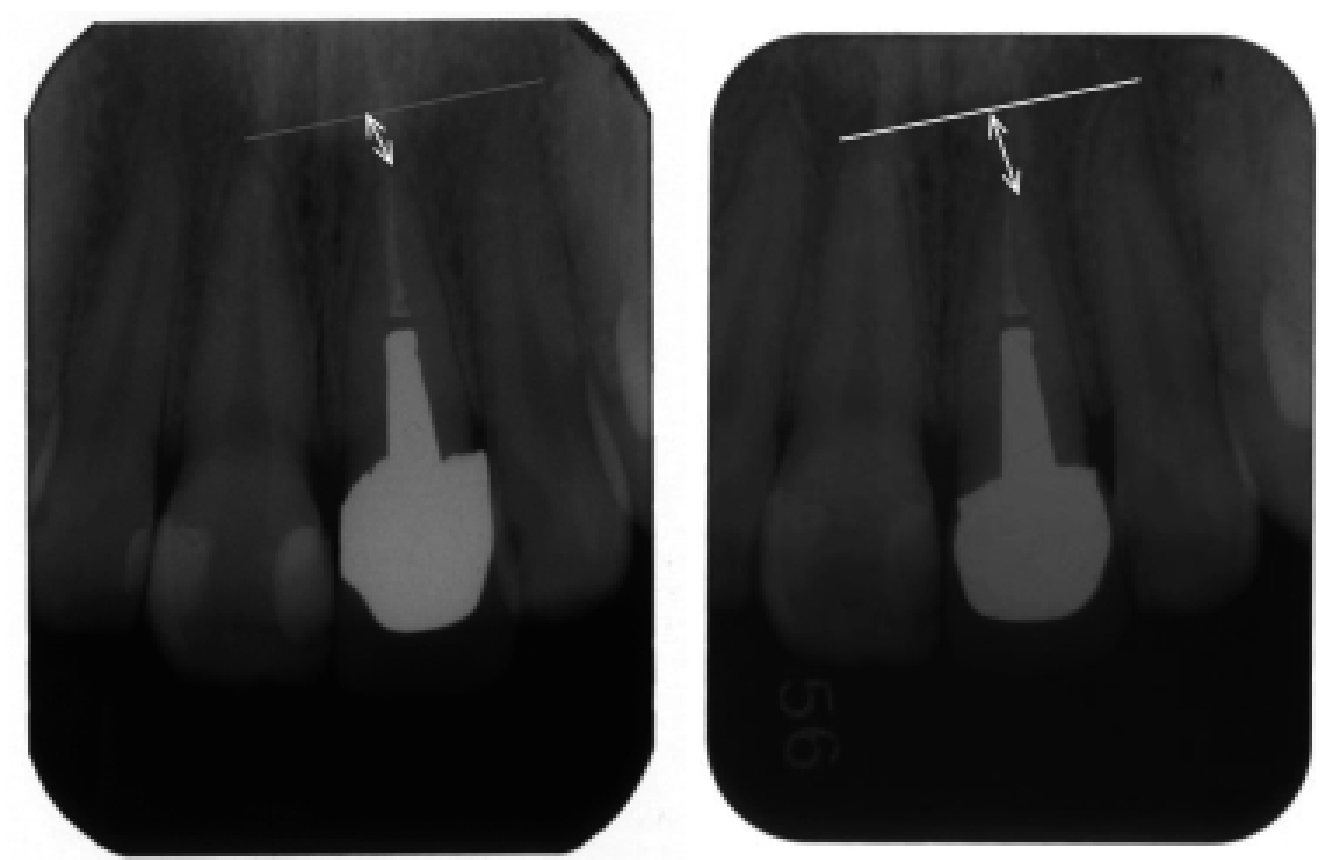

Fig.7 Radiographic evaluation. 


\section{Discussion}

A discrepancy in the labial gingival margin of the anterior maxillary area poses a major aesthetic problem (1). Orthodontic extrusion can be used to level and align the gingival margins and the crowns of the teeth to obtain aesthetic harmony. Ingber (2) managed soft tissue cosmetic deformities using orthodontic extrusion, but orthodontic extrusion can reverse the osseous architecture around the tooth being extruded. Kozlovsky et al. (6) described an orthodontic extrusion technique associated with CSF that prevented coronal migration of bone tissue and gingival tissue. Furthermore, Berglundh et al. (5) demonstrated that repeated fiberotomy led to pronounced recession of the gingival margin and extensive loss of the connective tissue attachment. We do not consider the coronal migration due to orthodontic extrusion desirable on the palatal half of the extruded tooth. Furthermore, facial fiberotomy would result in buccal recession (5). Therefore, partial CSF was performed on the palatal half of the root in our patients.

Many variations in orthodontic extrusion with CSF have been reported (3). Kozlovsky et al. (6) performed CSF on the extruded tooth every 2 weeks, while Carvalho et al. (7) activated fixed orthodontic appliances weekly with CSF, after which the extruded teeth were maintained in retention for 8 weeks. Levine et al. (8) allowed the severed gingival fibers to remain attached to the root joined in some manner with newly formed fibers from the soft tissue wound border for 3 weeks. We activated the fixed appliances every 2 weeks with CSF before allowing the formation of new fibers. No inflammation or coronal migration of the gingiva was observed in the palatal region during the period of extrusion.

Generally, the extruded teeth are splinted to adjacent teeth to prevent relapse. However, splinting tends to promote plaque accumulation, leading to caries and gingival inflammation. Simon et al. (9) recommended a minimum of 8 to 12 weeks of retention to stabilize the extruded tooth in its new position. We thus allowed 8 weeks of stabilization to prevent relapse. The position of the tooth and gingival margin has remained stable 2 years postoperatively.

Orthodontic extrusion also increases KG. Using tattoos in monkeys to mark the mucogingival junction and bottom of the clinical sulcus, Kajiyama et al. (10) evaluated the gingival movement associated with extrusion of the incisors. They found that the free gingiva moved about $90 \%$ and the attached gingiva about $80 \%$ of the extruded distance. Our results imply a similar periodontal tissue reaction. In this study, the KG remained stable for 2 years.

Orthodontic extrusion with palatal circumferential supracrestal fiberotomy was effective in improving the discrepancy in the labial gingival margin of maxillary incisors. Two years postoperatively, the positions of the teeth and gingival margins remained stable. However, this report consists solely of two case studies, and further clinical research is needed to confirm the results.

\section{References}

1. Kokick VG (2002) The role of orthodontics as an adjunct to periodontal therapy. In: Carranza's clinical periodontology, 9th ed, Newman MG, Takei HH, Carranza FA eds, W. B. Saunders, Philadelphia, 704-748.

2. Ingber JS (1989) Forced eruption: alteration of soft tissue cosmetic deformities. Int J Periodontics Restorative Dent 9, 416-425.

3. Pontoriero R, Clenza F Jr, Ricci G, Carnevale G (1987) Rapid extrusion with fiber resection: a combined orthodontic-periodontic treatment modality. Int J Periodontics Restorative Dent 5, 3143.

4. Zachrisson BU (2003) Orthodontics and periodontics. In: Clinical periodontology and implant dentistry, 4th ed, Lindhe J, Karring T, Lang NP eds, Munksgaard, Copenhagen, 744-776.

5. Berglundh T, Marinello CP, Lindhe J, Thilander B, Liljenberg B (1991) Periodontal tissue reaction to orthodontic extrusion. An experimental study in the dog. J Clin Periodontol 18, 330-336.

6. Kozlovsky A, Tal H, Lieberman M (1988) Forced eruption combined with gingival fiberotomy. A technique for clinical crown lengthening. J Clin Periodontol 15, 534-538.

7. Carvalho CV, Bauer FPF, Romito GA, Pannuti CM, Micheli GD (2006) Orthodontic extrusion with or without circumferential supracrestal fiberotomy and root planning. Int J Periodontics Restorative Dent 26, 87-93.

8. Levine HL, Stahl SS (1972) Repair following periodontal flap surgery with the retention of gingival fibers. J Periodontol 43, 99-103.

9. Simon JH, Kelly WH, Gordon DG, Ericksen GW (1978) Extrusion of endodontically treated teeth. J Am Dent Assoc 97, 17-23.

10. Kajiyama K, Murakami T, Yokota S (1993) Gingival reactions after experimentally induced extrusion of the upper incisors in monkeys. J Orthod Dentofac Orthop 104, 36-47. 